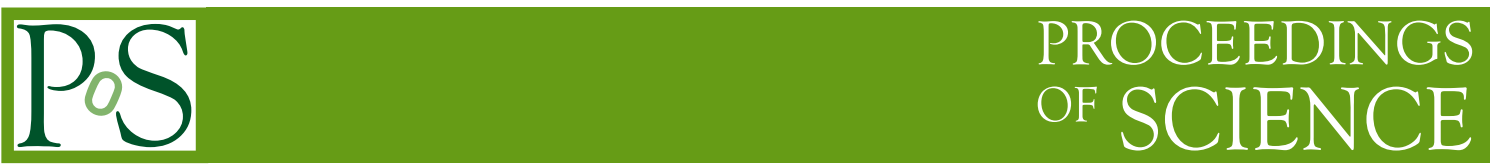

\title{
Upgrades of the CMS Outer Tracker for HL-LHC
}

\author{
Giacomo Sguazzoni ${ }^{* \dagger}$ \\ INFN Sezione di Firenze \\ E-mail: sguazzoniefi.infn.it
}

The LHC machine is planning an upgrade programme that will smoothly bring the luminosity up to or above $5 \times 10^{34} \mathrm{~cm}^{-2} \mathrm{~s}^{-1}$ sometime after 2020 , potentially allowing an integrated luminosity of $3000 \mathrm{fb}^{-1}$ to be achieved by the end of that decade. In this scenario, known as Phase-2, when the LHC will reach the High Luminosity (HL-LHC) phase, CMS will need a completely new Tracker detector, in order to fully exploit the demanding operating conditions and the delivered luminosity. The new Tracker should also have trigger capabilities. To achieve these goals, R\&D activities are ongoing to explore options and develop solutions that would allow the inclusion of tracking information at Level-1. The design choices for the CMS Outer Tracker upgrades are discussed along with some highlights of the R\&D activities.

The 23rd International Workshop on Vertex Detectors,

15-19 September 2014

Macha Lake, The Czech Republic

${ }^{*}$ Speaker.
†On behalf of the CMS Collaboration 


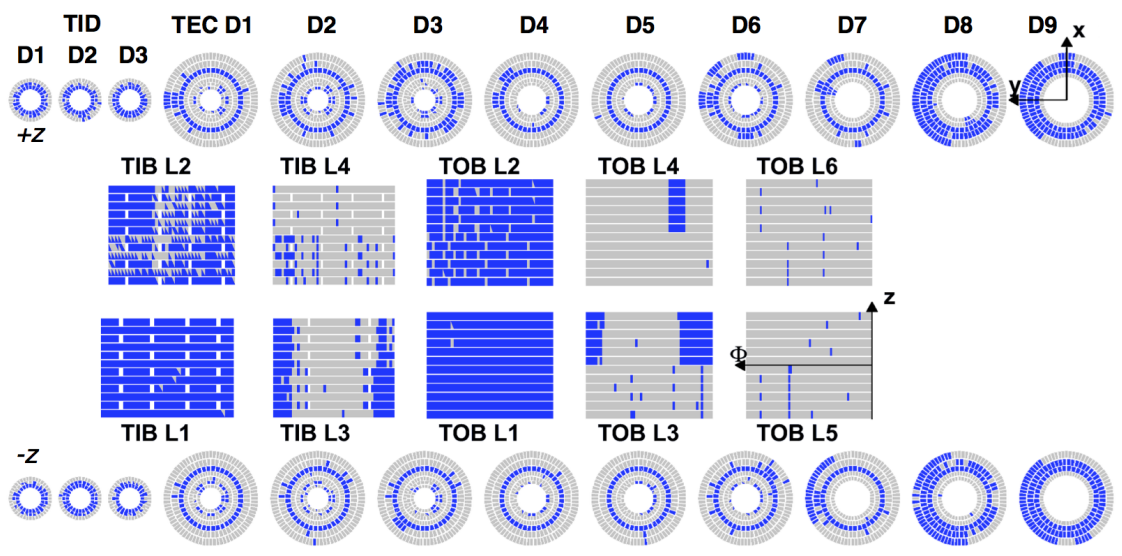

Figure 1: Map of non-functional modules (in blue) after the accumulation of an integrated luminosity of $1000 \mathrm{fb}^{-1}$, for the achievable minimum coolant temperature of $-20^{\circ} \mathrm{C}$.

\section{Introduction and motivations}

The present CMS Outer Tracker [1] was designed to operate without any loss of efficiency up to an integrated luminosity of $500 \mathrm{fb}^{-1}$ and an average pile-up (PU) of less than 50 collisions per bunch crossing.

During the High Luminosity phase of the LHC (HL-LHC), expected after 2020, the instantaneous luminosity will reach and then exceed $5 \times 10^{34} \mathrm{~cm}^{-2} \mathrm{~s}^{-1}$ with the aim of collecting $3000 \mathrm{fb}^{-1}$ of integrated luminosity in ten years. The present Outer Tracker and Phase-1 Pixel detector, which will be installed [2] at the end of 2016, would be unable to cope with even the first few years of operation of the HL-LHC and will therefore need to be replaced during the long shutdown (LS3) needed to install the machine components for the LHC upgrade.

The current Tracker will not be compliant with the significantly higher rate and longer latency of the upgraded trigger system that is crucial for operation at high luminosity. Moreover the increase of leakage current in the Outer Tracker sensors due to irradiation will be fatal. Figure 1 displays the simulated map of non-functional modules for the achievable minimum coolant temperature of $-20^{\circ} \mathrm{C}$ after $1000 \mathrm{fb}^{-1}$. Almost all the double-sided modules are no longer operational. A module is considered non-functional if the leakage current exceeds the maximum operating value of $3 \mathrm{~mA}$ that derives from the present power supply and cooling system limitations. The loss of hits on many layers of the tracker results in a significant degradation of track reconstruction performance, as seen in Fig. 2. The reduced efficiency and the increased fraction of ghost $\operatorname{tracks}^{1}$ have an unacceptable impact on the physics programme of CMS.

Despite the foreseen improvements, the muon and calorimeter-based lowest level trigger (Level1 or $L 1$ ) will not be able to withstand the rates due to the PU and the limited resolution: e.g., in the muon case, no transverse momentum $\left(p_{\mathrm{t}}\right)$ threshold is sufficiently effective in reducing the rate. The Tracker is then required to provide high- $p_{\mathrm{t}}$ tracks to the L1 system. Due to the increased L1 complexity the foreseen latency will be increased.

\footnotetext{
${ }^{1} \mathrm{~A}$ ghost track is a reconstructed track not unambiguously associated to a simulated track. The fraction of ghost reconstructed tracks is estimated with Monte Carlo simulation.
} 

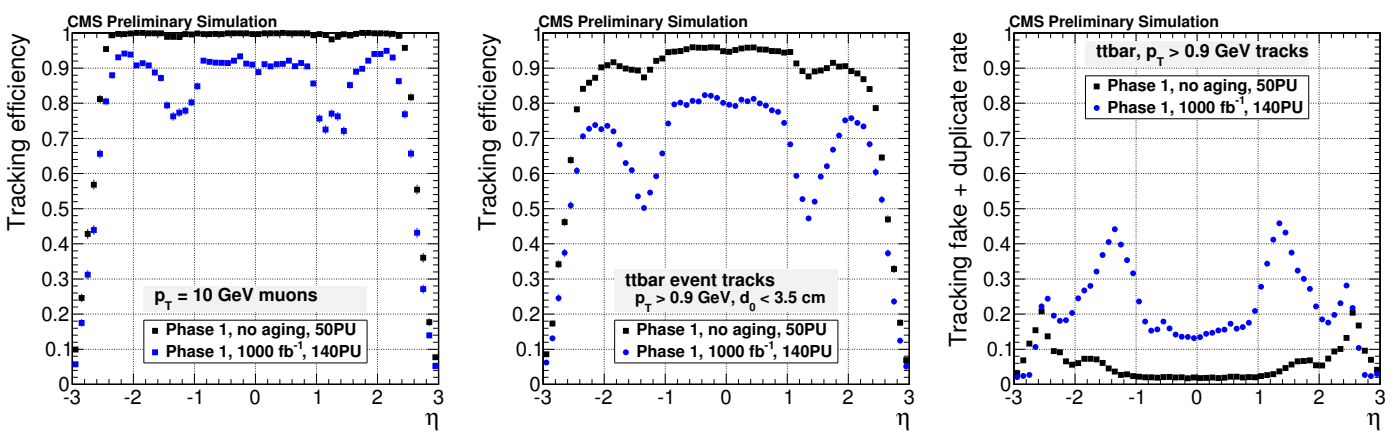

Figure 2: Efficiency as a function of pseudorapidity $(\eta)$ for $p_{\mathrm{t}}=10 \mathrm{GeV} / c$ muons (left) and for $t \bar{t}$ event tracks with $p_{\mathrm{t}}>0.9 \mathrm{GeV} / c$ and $d_{0}<3.5 \mathrm{~cm}$ (centre) for the Phase-1 detector before and after the Outer Tracker has been aged by an equivalent of $1000 \mathrm{fb}^{-1}$. Right: fraction of ghost reconstructed tracks for the same selection in $t \bar{t}$ events.

The main requirements for the Outer Tracker upgrade can be summarized as follows:

- radiation tolerance and cold $\left(-20^{\circ} \mathrm{C}\right)$ operation to make the Outer Tracker functional up to $3000 \mathrm{fb}^{-1}$.

- Increased granularity and optimized layout to keep the occupancy below O(1\%) up to PU 140 (possibly more) and to achieve a robust pattern recognition.

- Track trigger capabilities to contribute to the L1 trigger.

- Large readout bandwidth and deep front-end buffers to comply with the higher rate $(0.5-$ $1 \mathrm{MHz})$ and the long latency $(12.5 \mu \mathrm{s})$ of the ugraded L1 trigger system.

- Minimization and optimization of the passive material to reduce multiple scattering and radiation effects.

\section{Overview of the Phase-2 Outer Tracker Upgrade}

The Phase-2 Outer Tracker actually under study is sketched in Fig. 3. The L1 functionality is based on data reduction performed on the so-called $p_{\mathrm{t}}$-modules [7]. The $p_{\mathrm{t}}$-modules are composed of two closely-spaced silicon sensors read out by common front-end ASICs capable of correlating the hits from the two sensors, as sketched in Fig. 4; thanks to the strong magnetic field, hit pairs that form "stubs" compatible with particles above the chosen $p_{\mathrm{t}}$ threshold are selected and dispatched to the L1 trigger system at every bunch crossing. All other hits are stored in the front-end pipelines waiting to be read out when a trigger is received. A uniform $p_{\mathrm{t}}$ threshold throughout the tracking volume is achieved by tuning the acceptance window, programmable in the ASICs, and by choosing different sensor spacings across the tracker regions.

Two types of $p_{\mathrm{t}}$-modules are under development. "2S" modules (see Fig. 5) are composed of two strip sensors each approximately $10 \times 10 \mathrm{~cm}^{2}$ in area, with two rows of $\sim 5 \mathrm{~cm}$ AC-coupled strips with $90 \mu \mathrm{m}$ pitch. The sensors are mounted such that the strips are parallel. They will be used 


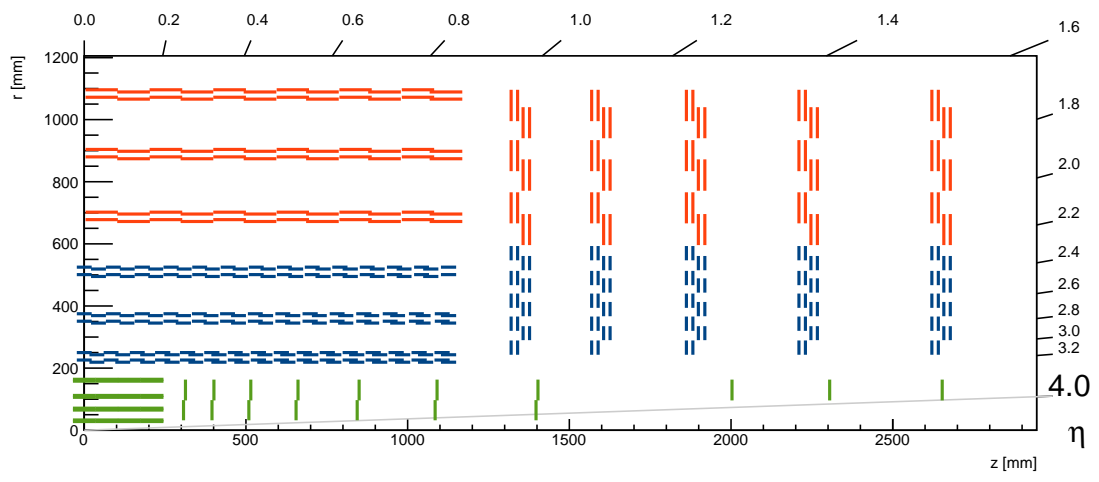

Figure 3: Sketch of one quarter of the Tracker Layout. Outer Tracker: blue lines correspond to PS modules, red lines to $2 \mathrm{~S}$ modules (see text). The Pixel detector with forward extension [3] is shown in green.

in the outer regions outside a radius of approximately $60 \mathrm{~cm}$ (in red in Fig. 3). "PS" modules (see Fig. 6) are composed of two sensors each approximately $5 \times 10 \mathrm{~cm}^{2}$ in area, one segmented into approximately $2.5 \mathrm{~cm}$ AC-coupled strips with $100 \mu \mathrm{m}$ pitch, and the other segmented in DC-coupled "macro-pixels" of size $100 \mu \mathrm{m} \times 1.5 \mathrm{~mm}$. PS modules are deployed in the radial range between $20 \mathrm{~cm}$ and $60 \mathrm{~cm}$, i.e. the inner region (blue in Fig. 3). The pixels precise measurements of the $z$ coordinate is crucial for primary vertex discrimination at L1 and for a robust pattern recognition. In both cases the sensors are connected to the readout hybrid and to the auxiliary electronics for powering and readout, all of which is integrated into the module assembly. One or more "service hybrids" carry a $5 \mathrm{~Gb} / \mathrm{s}$ data link, an optical converter, and the DC/DC converter that provides power to the module electronics.

The sensitive elements of the modules are planar silicon sensors, which will be exposed to particle fluences up to $1.5 \cdot 10^{15} \mathrm{neq} \cdot \mathrm{cm}^{-2}$, a factor of ten larger than the requirement for the present Tracker. A comprehensive programme has been carried out to identify suitable silicon materials $[4,5]$. To remove biases due to different vendors, only oxygen-rich $\left([\mathrm{O}]>8 \times 10^{16} \mathrm{~cm}^{-3}\right)$
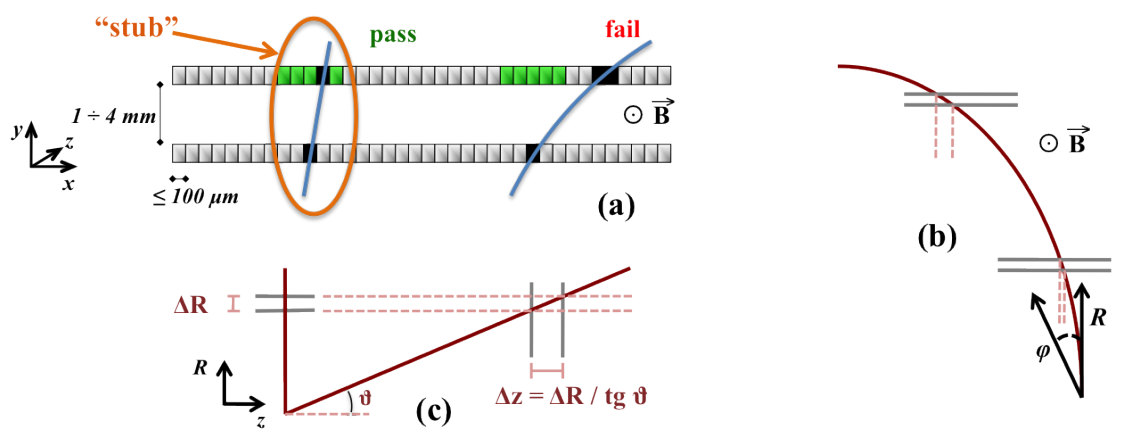

Figure 4: (a) Low- $p_{\mathrm{t}}$ hit rejection in closely-spaced sensors where the "selection window" to define an accepted "stub" is shown in green; (b) for constant spacing, the same $p_{\mathrm{t}}$ corresponds to a larger distance at larger radii; (c) for the end-cap disks, a larger sensor spacing is needed for a given discriminating power with respect to the barrel. 


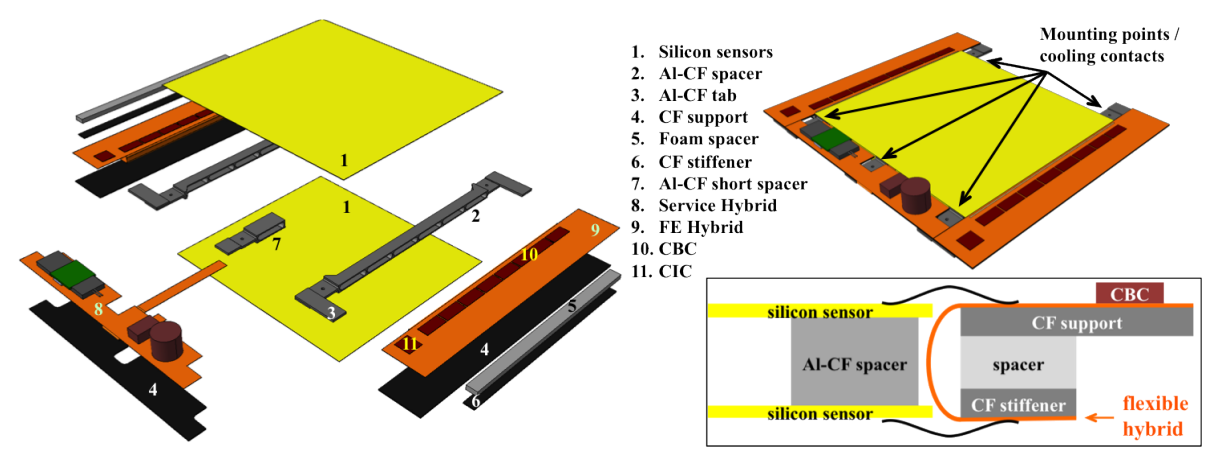

Figure 5: Exploded view of the $2 \mathrm{~S}$ module components (left), 3D view of the assembled module (upper right), and a sketch of the FE Hybrid folded around its support (lower right).

Hamamatsu Photonics samples have been studied, with a combination of:

- p-in-n or n-in-p implants;

- Float-zone, Magnetic Czochralski or Epitaxial substrates;

- $300,200,100,50 \mu \mathrm{m}$ active thicknesses.

The results of this campaign can be summarized as follows:

- n-in-p substrates are substantially more radiation tolerant [6] (either with p-spray or p-stop isolation) since charge collection degrades less with fluence, compared with p-in-n substrates (see Fig. 7); irradiated p-in-n sensors also show non-Gaussian noise that gives an irreducible and not negligible rate of fake hits;

- at highest dose, $200 \mu \mathrm{m}$ sensors have lower current and the same charge collection as $300 \mu \mathrm{m}$; moreover, at $600 \mathrm{~V}$ and $-20^{\circ} \mathrm{C}$, n-in-p $200 \mu \mathrm{m}$ thick substrates show a charge collection not

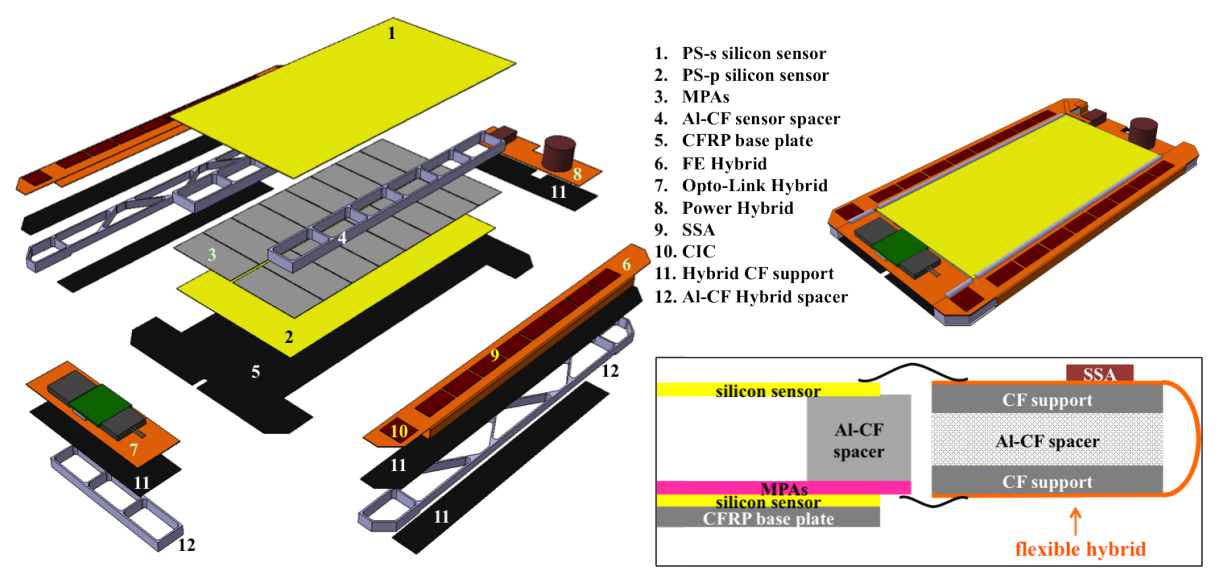

Figure 6: Exploded view of the PS module components (left), 3D view of the assembled module (upper right), sketch of the FE Hybrid folded around its support (lower right). 
degrading with the annealing time. It is therefore possible to exploit the annealing of the volume generated current, with no significant loss of signal.

The baseline layout of the Outer Tracker consists of 15508 detector modules (8424 2S and $7084 \mathrm{PS}$ ), with a total active surface of $218 \mathrm{~m}^{2}, 47.8 \mathrm{M}$ strips and $218 \mathrm{M}$ macro-pixels.

The electronics system, shown in Fig. 8, is designed to deliver trigger and L1 readout data with high efficiency up to a L1 trigger rate of $750 \mathrm{kHz}$, and to cope with latencies up to $12.5 \mu \mathrm{s}$.

At the front end, there are two FE Hybrids [9] interfacing to the two rows of strips of the silicon sensors in the $2 \mathrm{~S}$ module, or, in the case of the PS module, interfacing the strips with the MacroPixel-Sub-Assembly block (MaPSA), which integrates the pixelated sensor with its readout chips. The data generated by the FE chips are buffered, aggregated and formatted by the Concentrator IC (CIC) that feeds data to the Service Hybrid, which hosts all services to/from the counting room: data transfer $(4.8 \mathrm{~Gb} / \mathrm{s}$ bidirectional multi-mode optical link based on the low speed, low power grade version of the LP-GBT chip), low voltage powering (LV) and high voltage biasing (HV). The FE and Service Hybrids are multilayer high density flexible kapton circuits that are tightly folded by design (see Fig. 5 and 6) to ease connectivity to top and bottom sensor. Both $130 \mathrm{~nm}$ and $65 \mathrm{~nm}$ technologies are likely to be used for the FE ASICs in order to achieve adequate performance while minimizing development and production costs.

At the backend, the Data, Trigger \& Control Board (DTC) sends and receives data from multiple modules (typically 50-70 modules per card). This board is a custom development based on off-the-shelf commercial FPGAs and multi-channel optoelectronic transceivers. It processes three detector data streams: Timing and Control, DAQ and Trigger with a global usable bandwidth of $3.2 \mathrm{~Gb} / \mathrm{s}$ in each direction (the raw bandwidth, including Forward Error Correction, is $4.8 \mathrm{~Gb} / \mathrm{s}$ ).

The DTC board sends the stub stream to the L1 track finding system. Only about 5 to $10 \%$ of the roughly 10,000 stubs per bunch crossing actually belong to $p_{\mathrm{t}}>2 \mathrm{GeV} / c$ primary tracks. Three L1 Track Finding approaches are being pursued: in the Associative Memory (AM) [8] approach the pattern recognition is based on a massively parallel architecture that exploits preloaded

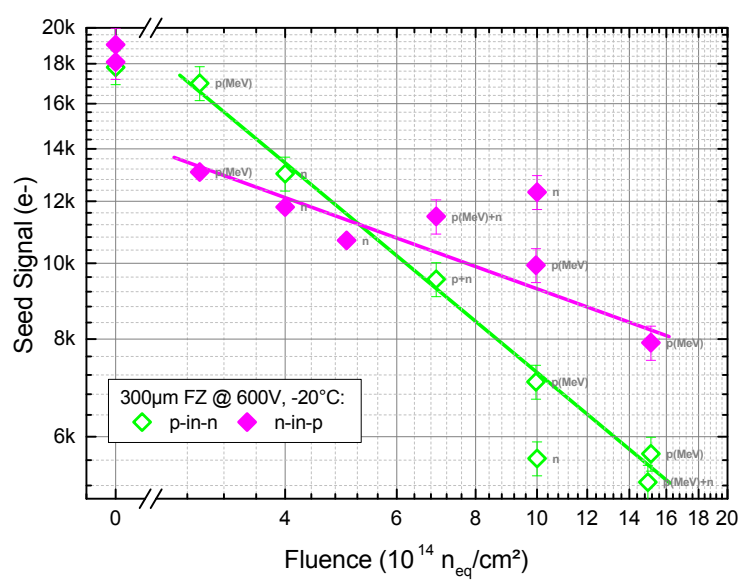

Figure 7: Charge collected on the seed strip (strip with the highest signal in a cluster) as a function of the fluence for $600 \mathrm{~V}$ biasing at $-20^{\circ} \mathrm{C}$ after short annealing (50h to $250 \mathrm{~h}$ ) at room temperature, for sensor thickness of $320 \mu \mathrm{m}$. Lines are drawn to guide the eye. 


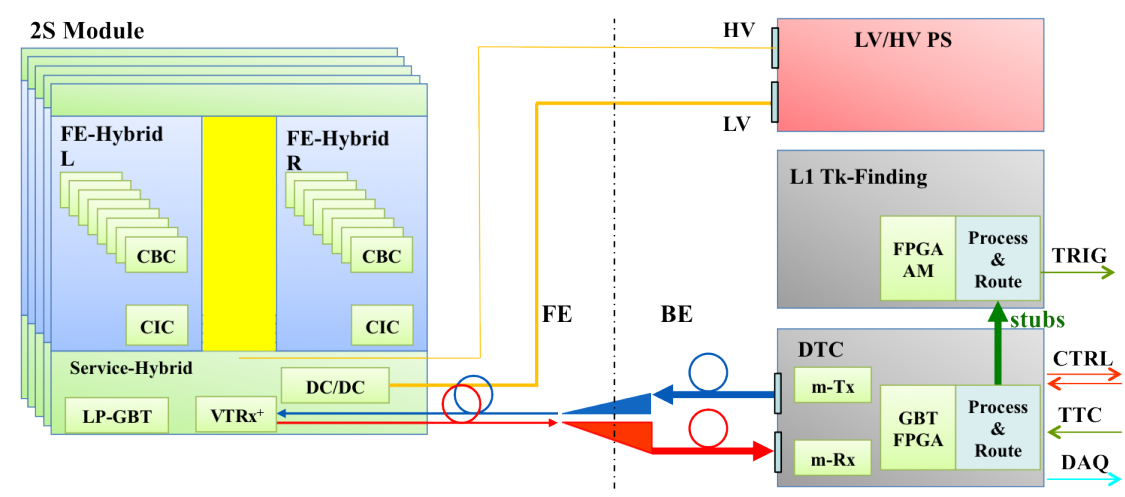

Figure 8: The electronics system block diagram for $2 \mathrm{~S}$ modules.

track patterns; a second approach is based on FPGAs implementing a road-based track search that attempts to find and join pairs of stubs in neighbouring layers (tracklets); the third approach, the Time Multiplexed Trigger (TMT), is similar to the current HLT architecture where the computing power is obtained by a large number of processors running in parallel and the crucial task is performed by the upstream event builder, i.e. a fast switch that routes the data coming from different parts of the detector to the same processor.

Power will be supplied to the FE at a voltage of $10-12 \mathrm{~V}$ whilst the HV supply will be designed to reach $-800 \mathrm{~V}$. The granularity of the distribution is still under consideration. Individual supply lines to each detector module would best match the Tracker modularity and alleviate the need for complex fail-safe redundancy schemes.

The mechanical concept of the Phase-2 Tracker (Fig. 9) is the same as for the current Tracker and consists of a support tube at the outermost radius. The $2 \mathrm{~S}$ modules that equip the TB2S (the three outermost barrel layers) are mounted onto 372 ladders, 12 modules each, then installed in a support wheel that consists of 4 vertical disks joined by cylinders at the inner and outer radii. The PS modules that equip the TBPS (the three innermost barrel layers) are glued directly onto 74 flat composite structures in which the cooling pipes are embedded. These plates are joined and supported by vertical rings to form the cylindrical layers. In the endcaps the modules are mounted on flat disks, which are split in half-disks, or "dees". Two disks are grouped to form one DoubleDisk; ten Double-Disk units will be produced, five for each end-cap or TEDD.
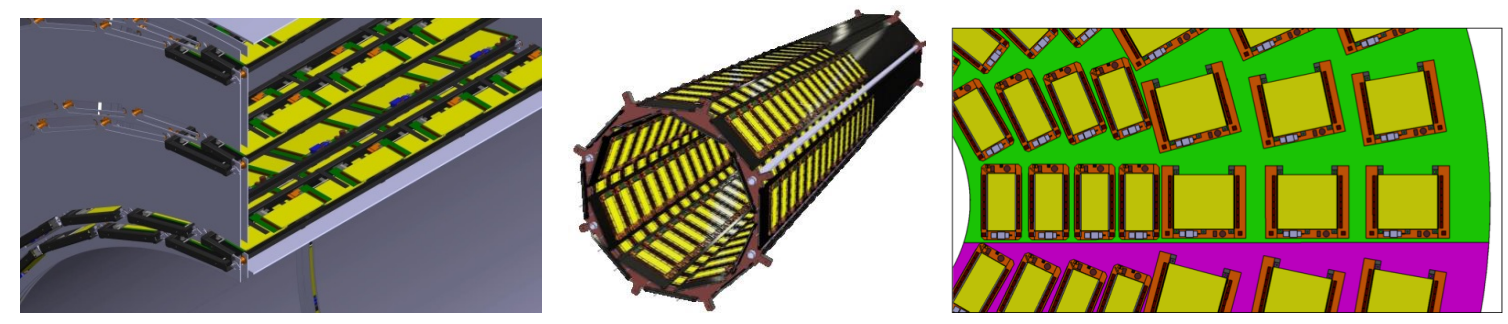

Figure 9: A detail of a TB2S wheel (left); a layer of the TBPS (centre); a detail of a TEDD disk (right). 

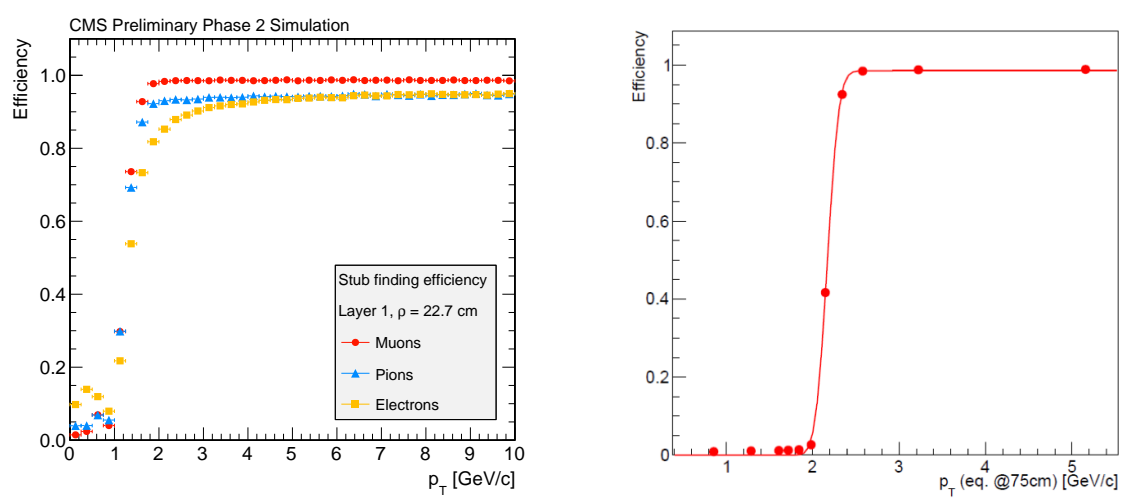

Figure 10: Stub finding efficiency: in the innermost barrel layer for muons, electrons and pions (left); measured using test beam data with $2 \mathrm{~S}$ module prototypes (right, see text).

The Tracker will be operated and maintained at $-20^{\circ} \mathrm{C}$ or lower using two-phase $\mathrm{CO}_{2}$ cooling. The total power dissipated by the Outer Tracker within the tracking volume is estimated to be around $\sim 90 \mathrm{~kW}$. Overall the material budget of the upgraded tracker is significantly reduced with respect to the current tracker with a $\sim 30 \%$ reduction (from $\sim 0.35 \mathrm{X}_{0}$ to $\sim 0.25 \mathrm{X}_{0}$ ) at $\eta \approx 0$ and a $\sim 50 \%$ reduction (from $\sim 1.6 \mathrm{X}_{0}$ to $\sim 0.8 \mathrm{X}_{0}$ ) at larger $|\eta|$ values.

\section{Performance}

The effectiveness of the $p_{\mathrm{t}}$-module concept is shown in Fig. 10, which shows the anticipated stub finding efficiency for simulated muons, electrons and pions and as measured from real testbeam data [10] with $2 \mathrm{~S}$ module prototypes, where different $p_{\mathrm{t}}$ values are emulated by setting an appropriate track incident angle assuming a radius of $75 \mathrm{~cm}$. The efficiency plateau is reached at $2 \mathrm{GeV} / c$ and the slightly lower efficiency for electrons and pions is due to interactions with detector material. These figures correspond to a hit over stub multiplicity ratio ranging from $\approx 6$ to $\approx 10$ in the barrel, to $\approx 7$ to $\approx 25$ in the endcaps.
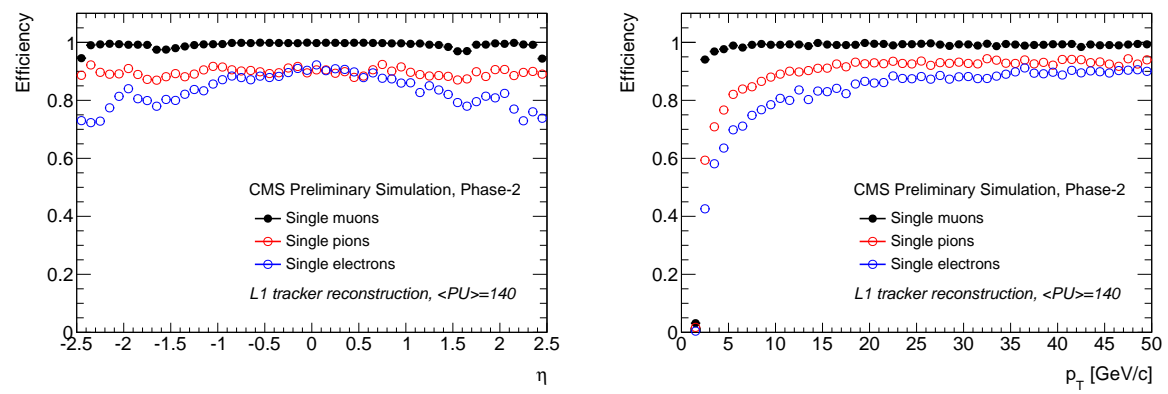

Figure 11: Efficiency for $\mathrm{L} 1$ track reconstruction as a function of $\eta$ (left) and $p_{\mathrm{t}}$ (right) for muons, pions, and electrons. 

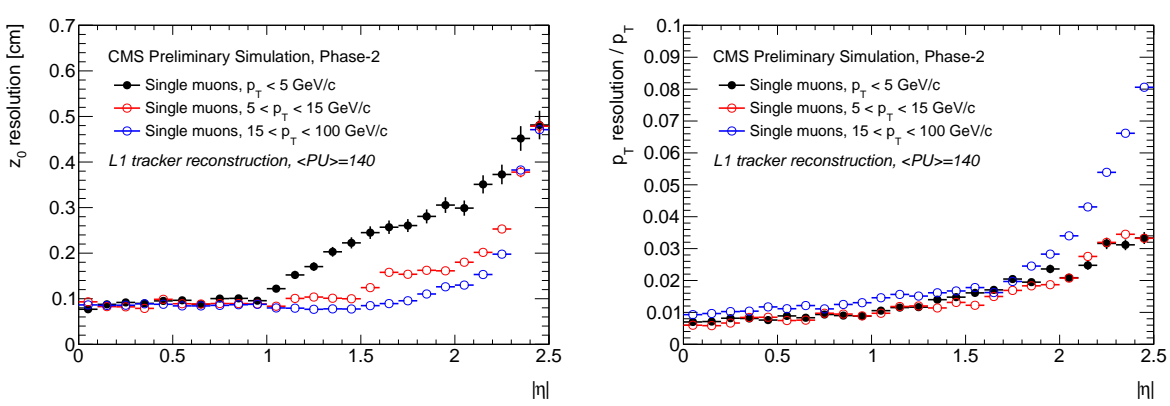

Figure 12: Resolution in $z_{0}$ and relative resolution in $p_{\mathrm{t}}$ for the $\mathrm{L} 1$ track reconstruction of single muons as a function of $|\eta|$ for different $p_{\mathrm{t}}$ ranges.

The L1 track finding efficiency, evaluated from samples of single muons, pions and electrons, uniformly distributed in $\phi, \eta$ and $p_{\mathrm{t}}$ and overlaid with an average of $140 \mathrm{PU}$ events, is shown in Fig. 11 for the tracklet algorithm. The AM-based pattern recognition shows similar features. The L1 track longitudinal impact parameter $\left(z_{0}\right)$ and $p_{\mathrm{t}}$ resolutions are shown for single muons in Fig. 12 as function of $|\eta|$ for three ranges of $p_{\mathrm{t}}$. The momentum resolution decreases as expected beyond $|\eta|>1.5$ due to the shorter effective lever arm. A $z_{0}$ resolution of approximately $\sim 1 \mathrm{~mm}$ is achieved in the central region, similar to the average separation of pile-up vertices.

Some highlights of the expected offline tracking performance [11] are shown in Fig. 13. The track reconstruction efficiency for the Phase-2 detector at $140 \mathrm{PU}$ for muons and $t \bar{t}$ events is as good as the Phase-1 efficiency at $50 \mathrm{PU}$ and extends the $|\eta|$ range by about 1.5 units thanks to the foreseen extension of the pixel detector [3]. The fraction of ghost reconstructed tracks is higher but still adequate for the CMS event reconstruction.

\section{Conclusions}

The Phase-2 Tracker upgrade for HL-LHC consists of a replacement of the whole tracker
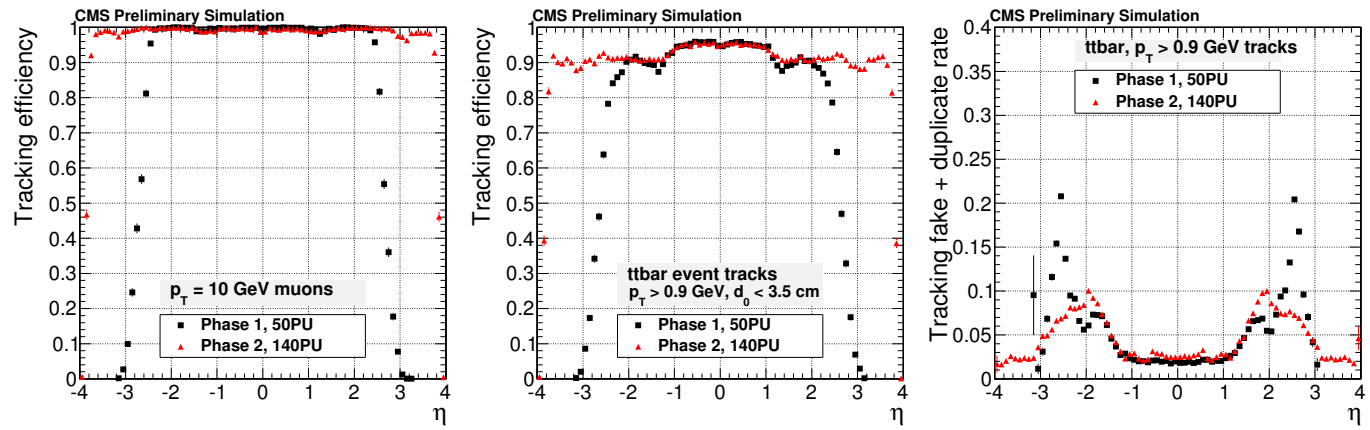

Figure 13: Track reconstruction efficiency as a function of $\eta$ for $p_{\mathrm{t}}=10 \mathrm{GeV} / c$ muons (left) and t⿱t events with $p_{\mathrm{t}}>0.9 \mathrm{GeV} / c$ and $d_{0}<3.5 \mathrm{~cm}$ (centre); on the right, for $\mathrm{t} \overline{\mathrm{t}}$ events, the fraction of ghost reconstructed tracks as a function of $\eta$. Comparison is shown between Phase-1 detector without aging at $50 \mathrm{PU}$ and the Phase-2 detector at 140 PU. 
detector. The development of the new Outer Tracker is well advanced. The innovative module design features on-board data reduction to comply with the required L1 track trigger capabilities. The detector layout is being optimized and finalized and several R\&D activities are ongoing on sensors, electronics and mechanics. The project will be described in a Technical Proposal expected in 2015, and the feasibility will be demonstrated in a Technical Design Report expected in 2017.

\section{References}

[1] The CMS Collaboration, The CMS experiment at the CERN LHC, JINST 3, S08004 (2008).

[2] The CMS Collaboration, CMS Technical Design Report for the Pixel Detector Upgrade, CERN-LHCC-2012-016.

[3] M. Musich, Upgrades of CMS inner detector for HL-LHC (pixels), these proceedings.

[4] K.-H. Hoffmann, Campaign to identify the future CMS tracker baseline, NIM A 658, 30-35, 2011.

[5] A. Dierlamm, Characterisation of silicon sensor materials and designs for the CMS Tracker Upgrade, PoS(VERTEX 2012) 016, 2012.

[6] http://rd50.web.cern.ch/rd50/

[7] G. Hall et al., 2-D PT module concept for the SLHC CMS tracker, JINST 5 C07012, 2010.

[8] F. Morsani et al., The AMchip: A VLSI associative memory for track finding, NIM A 315, 446, 1992.

[9] G. Blanchot et al., Hybrid circuit prototypes for the CMS Tracker upgrade front-end electronics, JINST 8 C12033, 2013.

[10] D. Braga et al., Beam test performance of the $2 S$ prototype module for the High Luminosity Upgrade of the CMS Strip Tracker, WIT 2014, 14-16 May 2014, to be published on JINST.

[11] The CMS Collaboration, Description and performance of track and primary-vertex reconstruction with the CMS tracker, JINST 9, P10009 (2014). 